\title{
Computational Fluid Dynamics Modelling and Experimental Study on a Single Silica Gel Type B
}

\author{
John White \\ School of Mechanical Engineering, University of Birmingham, Birmingham B152TT, UK \\ Correspondence should be addressed to John White, jxw998@bham.ac.uk
}

Received 6 December 2011; Revised 23 January 2012; Accepted 24 January 2012

Academic Editor: Guan Heng Yeoh

Copyright ( 2012 John White. This is an open access article distributed under the Creative Commons Attribution License, which permits unrestricted use, distribution, and reproduction in any medium, provided the original work is properly cited.

The application of computational fluid dynamics (CFDs) in the area of porous media and adsorption cooling system is becoming more practical due to the significant improvement in computer power. The results from previous studies have shown that CFD can be useful tool for predicting the water vapour flow pattern, temperature, heat transfer and flow velocity and adsorption rate. This paper investigates the effect of silica gel granular size on the water adsorption rate using computational fluid dynamics and gravimetric experimental (TGA) method.

\section{Introduction}

The adsorption properties of silica gel have been studied for many years as silica gel is used in many industries including adsorption cooling systems. Past research has shown experimentally that the adsorpitivity of silica gel granules depends on their size. The purpose of this work was to study the effect of granule size on the adsorption properties of silica gel by using CFD and comparing the simulation results to those obtained experimentally. Computational fluid dynamics (CFD) is a field that has developed over the years because of the new development of computers and CFD simulation. CFD is also used a simulation tool in refrigeration and air conditioning industry. In this paper CFD simulation has been used for modelling of heat and mass transfer in an adsorption tube with silica. One of the questions in CFD modelling is can this type of technology be used as an alternative to experimental data gathering. For this hypothesis a confirmation of CFD data against experimental data is desired. In this paper a 3D silica gel and tube-structured model have been developed for this purpose. Data will be gathered from experimental study, an identical CFD model. This data will be compared to experimental and CFD results. The effects of silica gel size and properties have been studied using the porous media approaches by application of modified Navier-Stokes equations and the continuity equation. In this set of equations, an additional term is used describing the porosity distribution in order to take into account the porous media packing geometry. To design an efficient adsorber bed, flow and temperature information is essential. Studies of fluid dynamics and heat transfer in adsorber beds date back to the early twentieth century [1] The early investigation of flow in porous media packed beds provided mainly such bulk information as pressure drop correlations. For example, Ergun [2] suggested a pressure loss equation, in which parameters like porous media particle diameter and fluid physical properties were used to correlate the pressure drop. Similar correlations were proposed by Molerus [3].

These three empirical correlations rather well predict pressure loss for flow in adsorber beds but provide nothing more about local flow fields of our adsorption/desorption on porous media. On the other hand, of the many investigations of heat transfer in adsorber beds in the last several decades effective parameter methods were applied to predict temperature distribution $[4,5]$. Computational fluid dynamics (CFDs) provide an innovative approach to model and analysing the local flow and the effect of silica gel size on adsorption performance of a packed bed. Numerical simulation of porous media local flow and heat transfer based on CFD technique has increasingly been reported in recent years in fields of packed bed flow and heat transfer 
modelling [6]. In this work, a finite volume formulation based on the Chimera meshing technique is used to simulate the local flow and heat transfer in a silica gel packed bed, which contains 120 randomly packed spheres.

\section{Experimental Apparatus Description}

There are numerous methods used for measuring the adsorption uptake of an adsorbent, and the most widely reported procedures are the gravimetric method and the volumetric method. The experimental apparatus used in this paper is the gravimetric method (TGA) developed by Micromeritics instrument corporation; this system is designed to measure adsorption/desorption of porous media materials (see Figure 2 for a schematic layout of the system instruments).

2.1. Experimental Setup and Procedure. The thermogravimetric assembly (TGA) method is used for isotherm, kinetics adsorption experiments due to the high accuracy, ease of control of the pressure and temperature of the experiment. TGA gives a direct measurement of the quantity of adsorbate adsorbed throughout the uptake process. A microbalance device is used where the adsorbent mass is measured as a function of temperature and time. In the TGA, the sample container is suspended on an extension wire that is connected to the microbalance (see Figure 3).

The microbalance measures the weight of sample, and it can detect weight changes of the sample in a very short time. Its measurement ranges are between 0 and $150 \mathrm{mg}$ with readability up to $0.1 \mu \mathrm{g}$. The control software screen shot displays control of the functions of the microfurnace, balance, and thermocouple; this runs on a computer connected to the TGA's thermal gas analysis station. During the experiment, time, weight, and temperature are recorded continuously at defined intervals and the data are stored on the computer.

Silica gel granules sample used in the experiment was Fuji type B size $3.5 \mathrm{~mm}$ mesh 10-40 (see Table 1 for thermophysical properties of this silica gel).

In Figure 4 you can see a single silica gel size $3 \mathrm{~mm}$ type $\mathrm{B}$; the purpose of testing one silica gel adsorption/desorption was to generate experimental data to be used in a CFD module. The advantage of studying a single granule of silica gel is very useful when designing a $3 \mathrm{D}$ adsorption bed simulation prediction module.

\section{GTA Experimental Results}

The adsorption isotherms of the Fuji Davison silica gel water systems are known to exhibit zero hysteresis. Hence in the spirit of confirming the reliability of our analyzer, experiments are carried out from low vapour exposure to high vapour exposure (low-high process) and then from high coverage back to low exposure (high-low process). Figure 5 shows a typical adsorption isotherm plot for water vapour adsorption on the Fuji Davison type B size $3.5 \mathrm{~mm}$ silica gel (specific surface area is around $800 \mathrm{~m}^{2} \mathrm{~g}^{-1}$ ). Figure 6 presents the water vapour adsorption uptake on the type $B$ silica
TABLe 1: Thermophysical properties of this silica gel. A porous material had to be defined for the adsorbent used in the simulation. This data was taken from the experiment Type B data was created with a density of $730 \mathrm{~kg} / \mathrm{m}^{3}$, for type B and a density of $1000 \mathrm{~kg} / \mathrm{m}^{3}$ and a heat capacity of $0.921(\mathrm{KJ} / \mathrm{kg} \mathrm{K})$ and a thermal conductivity of $0.174(\mathrm{~W} / \mathrm{m} \mathrm{K})$ (see Table 1$)$.

\begin{tabular}{lc}
\hline & Type B \\
\hline Specific surface area $\left(\mathrm{m}^{2} / \mathrm{g}\right)$ & 450 \\
Porous volume $(\mathrm{mL} / \mathrm{g})$ & 0.85 \\
Average pore diameter $(\mathrm{A})$ & 22 \\
Apparent density $\left(\mathrm{kg} / \mathrm{m}^{3}\right)$ & 730 \\
$\mathrm{pH}$ value & 5.0 \\
Water content $($ wt. $\%)$ & $<2.0$ \\
Specific heat capacity $(\mathrm{kJ} / \mathrm{kg} \mathrm{K})$ & 0.921 \\
Thermal conductivity $(\mathrm{W} / \mathrm{m} \mathrm{K})$ & 0.174 \\
Mesh size & $10-40$ \\
\hline
\end{tabular}

TABLE 2: Thermophysical properties used in CFD simulation. The adsorption performance of silica gel is influenced by the physical properties such as surface area, pore size and pore volume and pore distribution, porosity, and density of the silica gel see Table 2 .

\begin{tabular}{lc}
\hline Material & Water \\
\hline Density, $\rho\left(\mathrm{kg} / \mathrm{m}^{3}\right)$ & 1000 \\
Specific heat capacity, $C_{p}(\mathrm{~J} / \mathrm{kg} \mathrm{K})$ & 4200 \\
Thermal conductivity, $k(\mathrm{~W} / \mathrm{mK})$ & 0.61 \\
Dynamic viscosity, $\mu(\mathrm{kg} / \mathrm{ms}) \times 10^{-3}$ & 0.96172 \\
\hline
\end{tabular}

gel size $5 \mathrm{~mm}$ following the low-high process at $25^{\circ} \mathrm{C}$. The adsorption temperature fluctuation is about $0.5^{\circ} \mathrm{C}$ which is controlled by the microcomputer controller (see Figure 1, pictorial view of the used TGA instrument).

3.1. The Isotherm Plot for Silica Gel Size $3.5 \mathrm{~mm}$. The adsorption/desorption Isotherm plot for silica gel size $3.5 \mathrm{~mm}$ for temperature $25^{\circ} \mathrm{C}$ in Figure 5 shows a typical water sorption result from a DVS experiment. The isotherm plot adsorption kinetic data shows the change in mass and humidity as over a function of time. From the kinetic results, the rate of water uptake and water diffusion coefficients can be determined. The equilibrium mass values at the end of each humidity step were used to calculate the sorption and desorption isotherms. The difference in water vapour uptake between the sorption and desorption isotherms is called the hysteresis.

3.2. The Isotherm Plot for Silica Gel Size $5 \mathrm{~mm}$. In Figure 6 the Isotherm plot is for silica gel size $5 \mathrm{~mm}$; the plot begins at water performance near zero and 1 . The shape and location of the isotherm hysteresis give you information about the sorption method and sample porosity. Temperature of water vapour in the adsorbent sample chamber is at a constant $25^{\circ} \mathrm{C}$ silica gel size $5 \mathrm{~mm}$. The isotherm plot will be used to assist in determining drying rates and optimal endpoints. The isotherm also shows whether the silica gel exhibits 

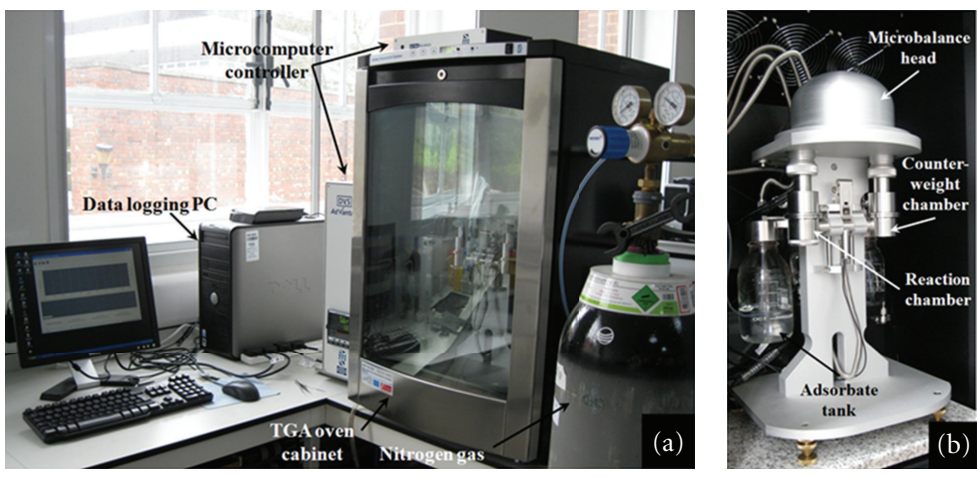

FIGURE 1: Pictorial view of the used TGA instrument; this system measures the change in weight of a silica gel sample as it is heated for desorption of water, cooled for the adsorption of water vapour, or held at a constant temperature.

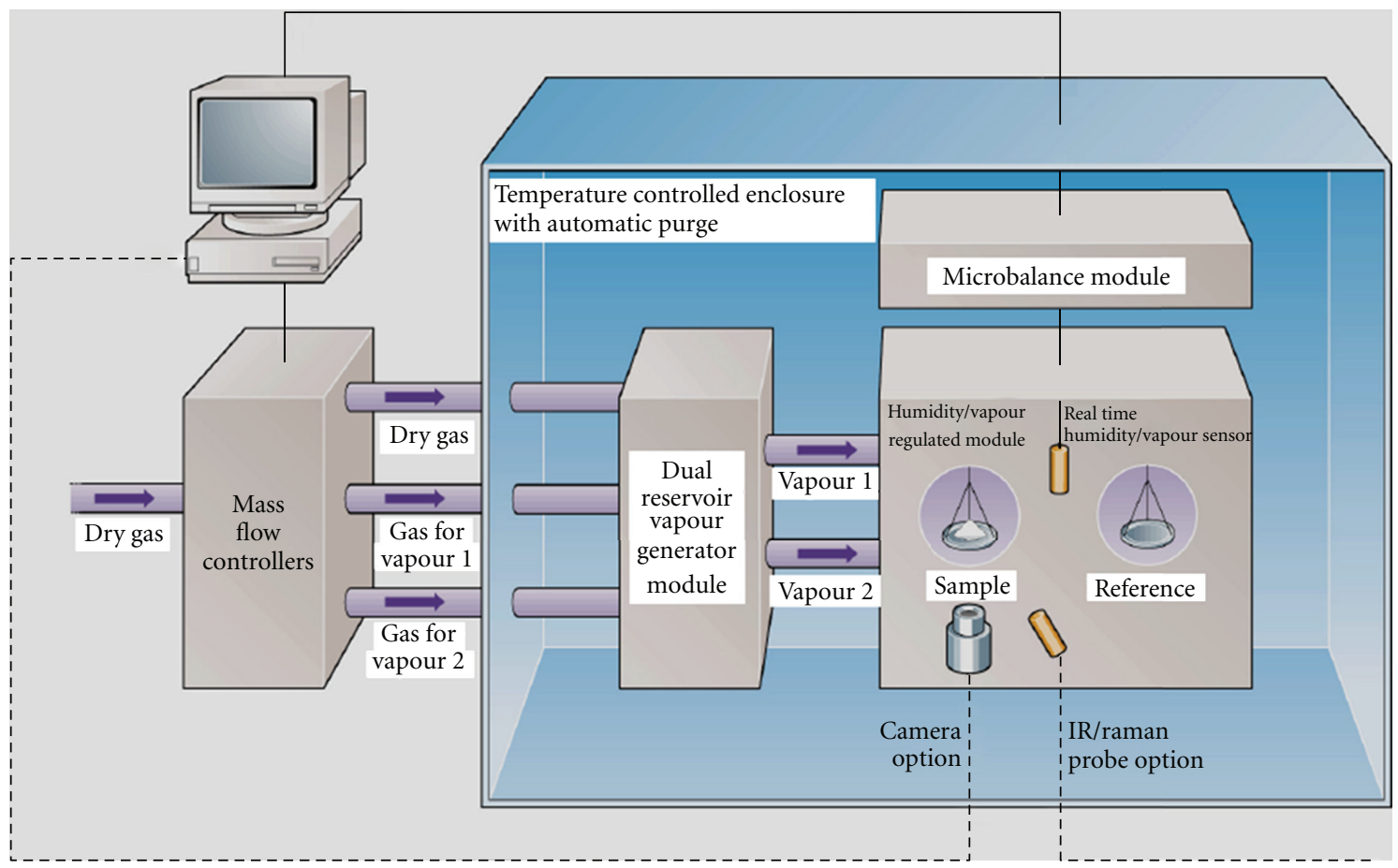

FIgUre 2: Schematic of the TGA instrument.

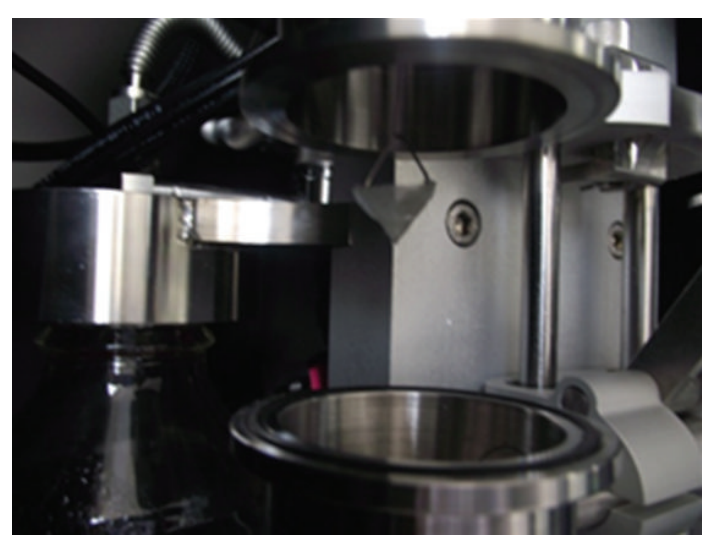

(a)

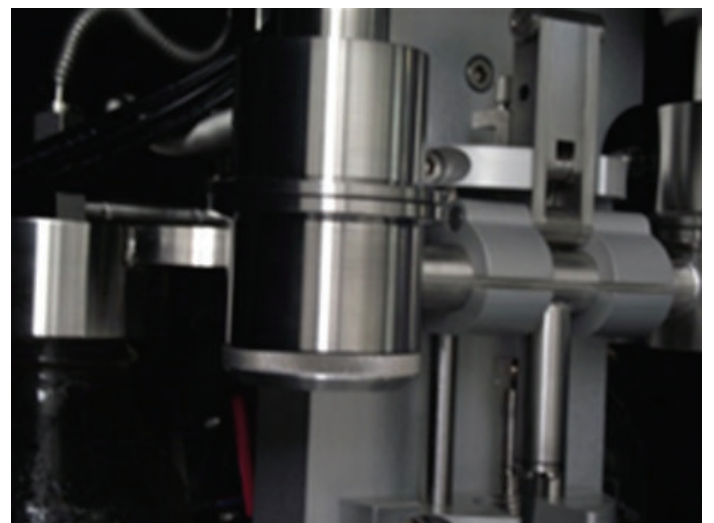

(b)

FIGURE 3: Sample container suspended on an extension wire. 


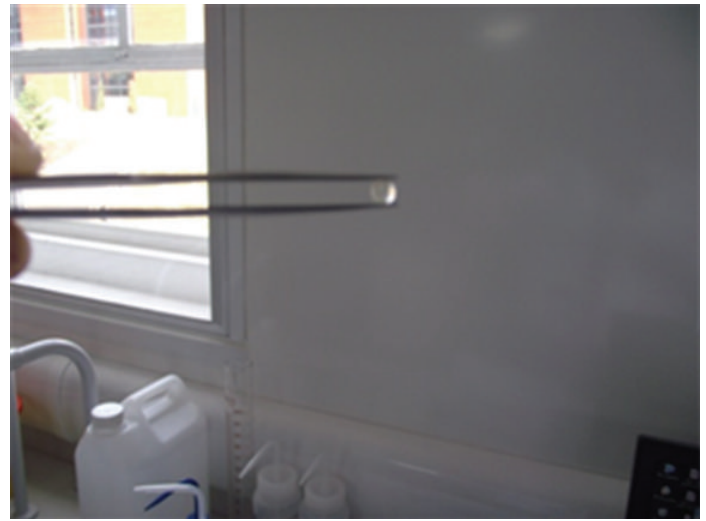

(a)

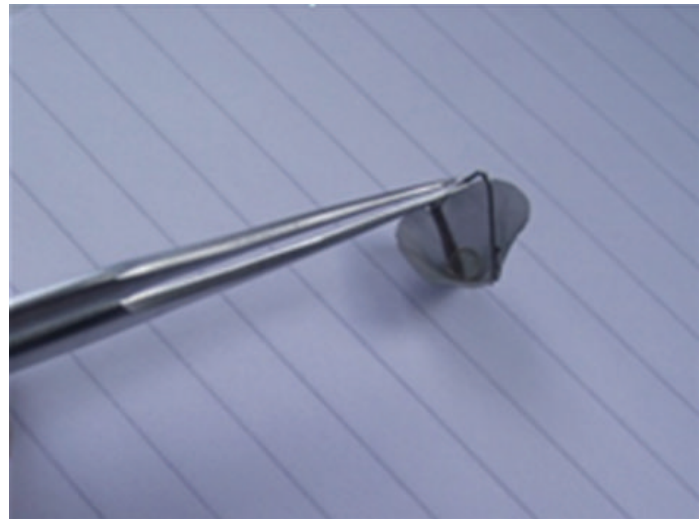

(b)

Figure 4: One silica gel size $3 \mathrm{~mm}$ type B.

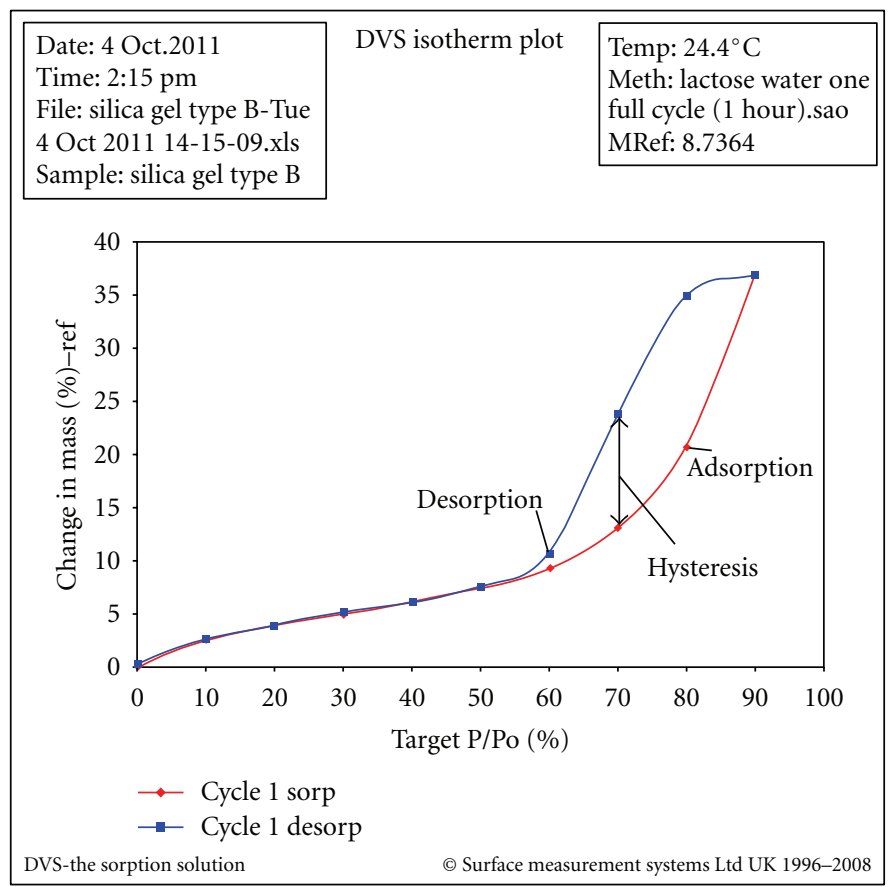

Figure 5: Moisture sorption behaviour of silica gel type B size $3.5 \mathrm{~mm}$ at $25^{\circ} \mathrm{C} 1$-cycle experiment. Figure 5 shows two isotherms, one obtained by wetting a sample from a dry state and the other obtained by drying a sample from wet state. Water sorption (red) and desorption (blue) isotherms at $25^{\circ} \mathrm{C}$ measured on silica gel. The moisture content at each water activity is higher during desorption (drying from high moisture content) than adsorption (wetting from low moisture content).

hysteresis and what impact it will have on water activity after drying to a given moisture content drying curve for silica gel type size $5 \mathrm{~mm}$. Figure 6 shows two isotherms, one obtained by wetting the silica gel sample from a dry state and the other obtained by drying the silica gel sample from wet state. The water sorption is shown (red) and desorption is shown (blue); isotherms temperature is at $25^{\circ} \mathrm{C}$. In this Isotherm plot the moisture content at each water activity is higher during desorption (drying from high moisture content) than adsorption (wetting from low moisture content). The isotherm plot represents the limits or bounding isotherms since they begin at water performance near zero and one. The shape of the isotherm hysteresis gives you information about the sorption mechanism and sample porosity of the $5 \mathrm{~mm}$ silica gel.

The shape and location of the isotherm hysteresis for silica gel type $B$ size $5 \mathrm{~mm}$ temperature of water vapour in the adsorbent sample chamber is at a constant $25^{\circ} \mathrm{C}$ silica gel size $5 \mathrm{~mm}$.

3.3. Physical Properties. The adsorption performance of silica gel is influenced by the physical properties such as surface area, pore size and pore volume and pore distribution, porosity, and density. 


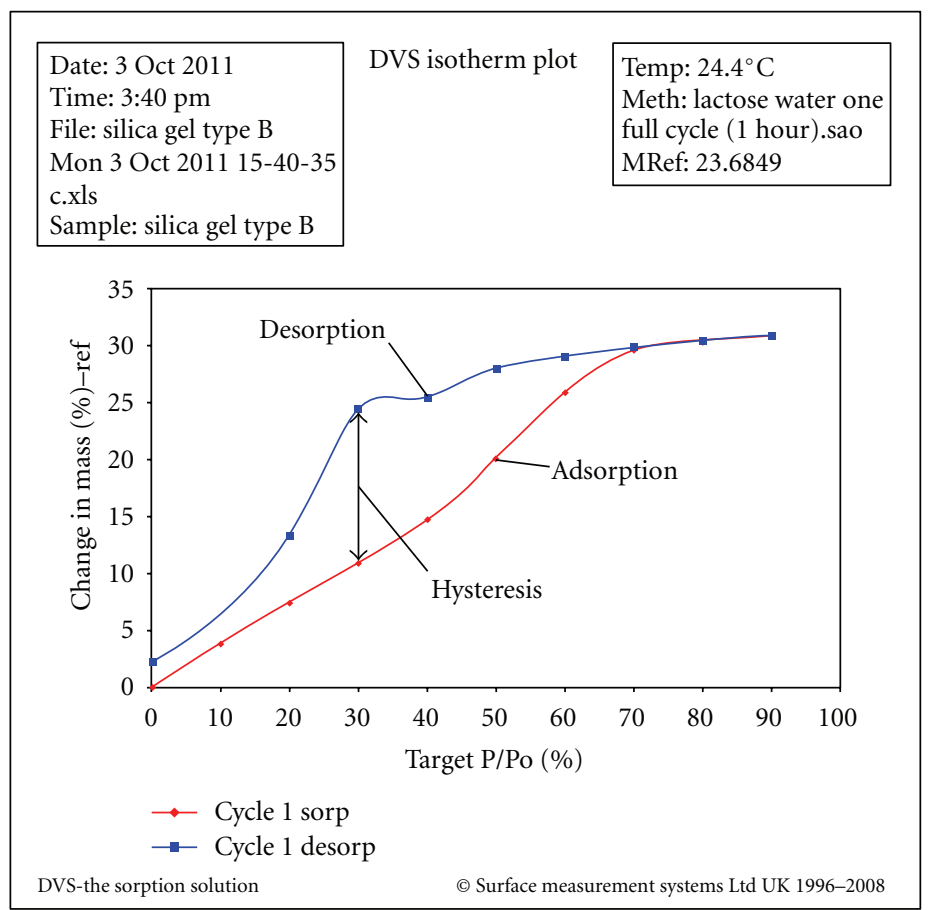

FIgURE 6: Moisture sorption behaviour of silica gel type B size $5 \mathrm{~mm}$ at $25^{\circ} \mathrm{C} 1$-cycle experiment.

To help identify the desorption of different species, derivative curves were also produced; the results obtained from a sample of silica gel taken directly from the TGA instrument are shown in Figures 5 and 6.

\section{Computational Fluid Dynamics}

In this study an integrated CFD model was developed to simulate the adsorption dynamics of water vapour of silica gel granules in an adsorption tube by using the SolidWorks.

Flow Simulation module consisted of two modes of operation, the water vapour adsorption and the desorption mode. In each mode, the water vapour flow profile surrounding a single silica gel granules was determined by solving the Navier-Stokes equations, and the resulting velocity profile was regarded time invariant and stored for later use. Also, the time-dependent mass transfer both outside and inside the porous silica gel due to its adsorpitivity was simulated through a user defined function developed to solve the Brunauer, Emmett, and Teller (BET) equation [7] for both adsorption and desorption processes. The developed simulation model was used to determine the adsorption capacity of two different sizes of silica gel granules, namely, $3.5 \mathrm{~mm}$ and $5 \mathrm{~mm}$ as a function of time at different operating temperatures. The simulation results were compared with experimental data and found to agree.

4.1. Geometry and Analysis. Geometrical modelling was one of the most critical stages in the CFD simulation; correct definition of the geometry provides a more practical state for the simulation, and the technique used for constructing the geometry will ensure the feasibility of generating a mesh good enough to capture all of the phenomena involved in the problem.

4.2. Boundary Conditions. The boundary conditions determine the flow and thermal variables on the boundaries of the physical model. There are a number of classifications of boundary conditions:

(1) flow inlet and exit boundaries: pressure inlet, velocity inlet, pressure outlet,

(2) wall, repeating, and limit boundaries: wall, symmetry,

(3) internal fluid, solid,

(4) internal face boundaries: porous, wall, interior.

In our model we use a velocity inlet at the flow inlet of the adsorption bed; this boundary condition defines a flow velocity at the inlet of the bed. The flow exit boundary is defined as a pressure outlet; the outlet pressure is defined as atmospheric pressure. The bed and packing interior are defined as boundaries. The wall boundaries separate the fluid zone, vapour, in between the silica gel particles from the wall zones $[5,8-11]$. With the determination of the boundary conditions, the physical model has been defined and a numerical solution was provided. It was then necessary to determine how the solution will be established. This was done by setting the iteration parameters. With all boundary conditions defined, a number of additional parameters and solving schemes where selected. An initial condition was assigned to the model and was used to help speed the convergence of the computation. 


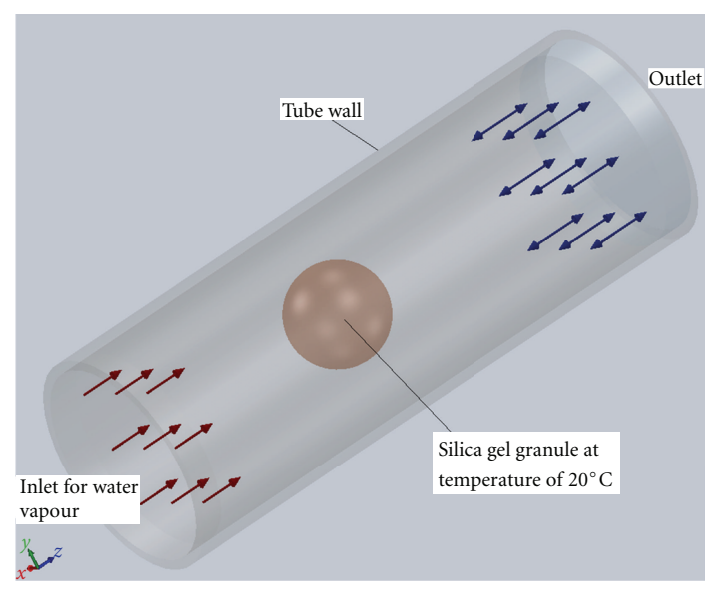

FIGURE 7: One silica gel in a tube geometry used for validation of CFD against experimental work.

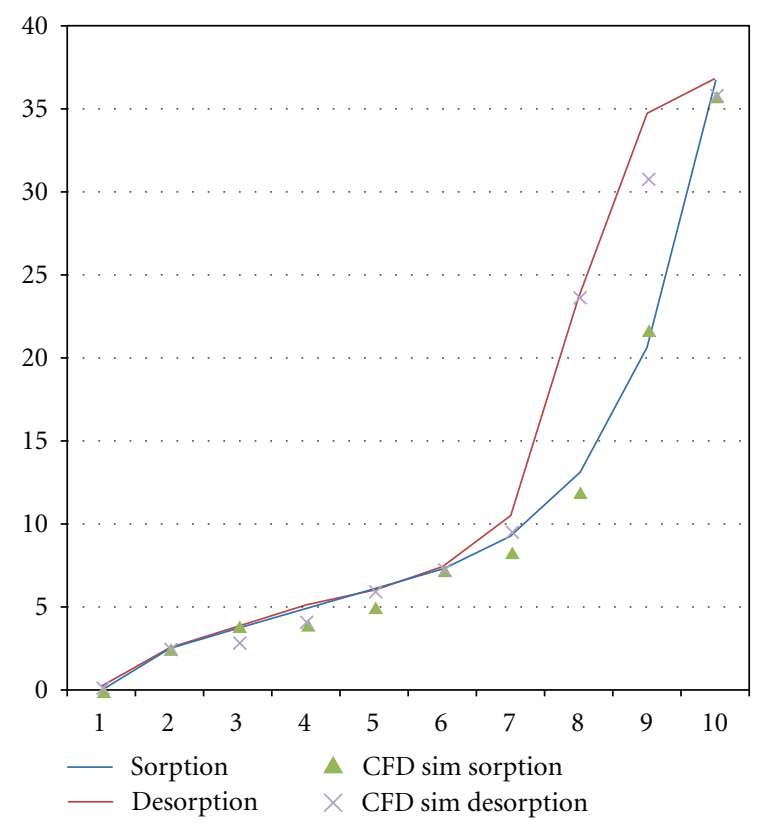

FIGURE 8: Adsorption and desorption curves for silica gel granules size $3.5 \mathrm{~mm}$.

The computation is an iterative process that solves the governing equations for flow and energy in each simulated cell.

4.3. Modelling of Vapour Flow in a Single Silica Gel Particle. The diffusion equation in the silica gel particle is as a first introduction to CFD a simple model was created the model represented one silica granule in a tube as shown in Figure 7 the flow inlet and flow outlet.

4.4. Porous Media Simulations. A porous material had to be defined for the adsorbent used in the simulation. This data was taken from the experiment Type B; data was created with a density of $730 \mathrm{~kg} / \mathrm{m}^{3}$ for type B and a density of

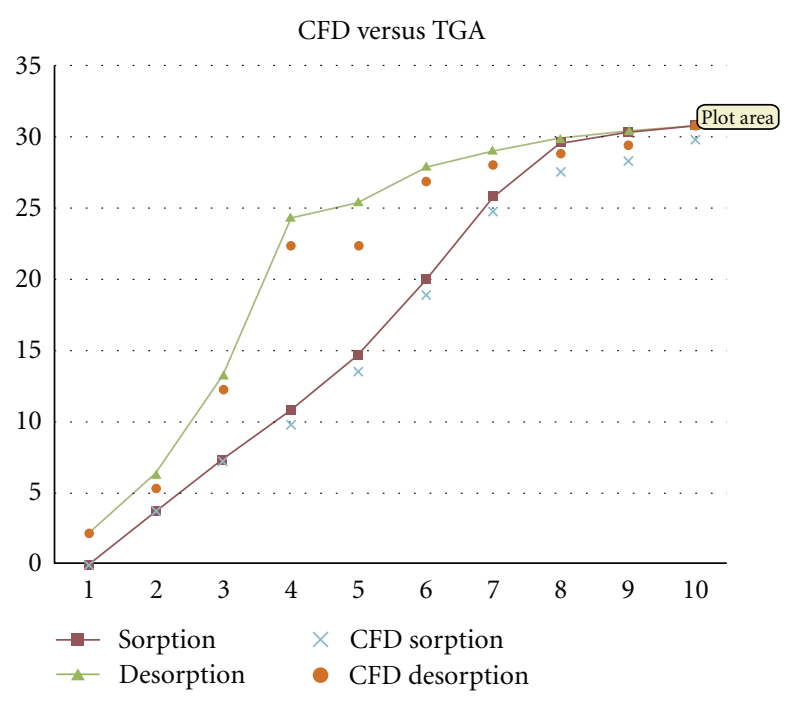

FIGURE 9: Adsorption and desorption curves for silica gel granules size $5 \mathrm{~mm}$.

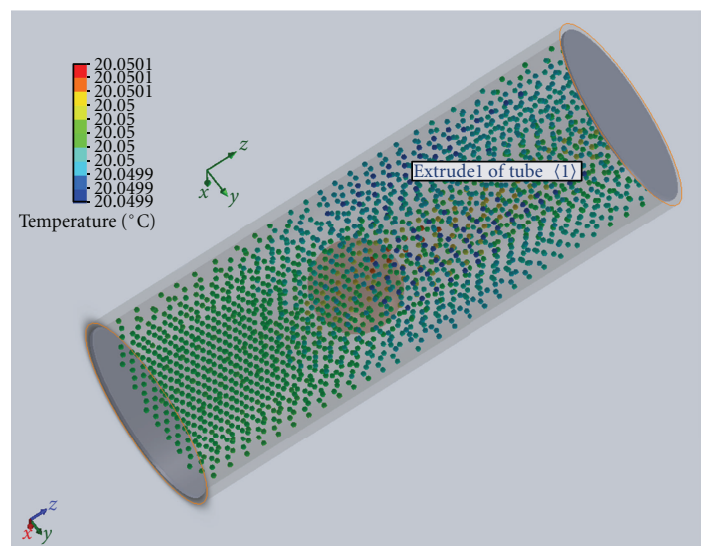

FIGURE 10: Simulation of water vapour adsorption on porous silica gel.

$800 \mathrm{~kg} / \mathrm{m}^{3}$ and a heat capacity of $0.921 \mathrm{~J} / \mathrm{kg} \mathrm{K}$ and a thermal conductivity of $0.174 \mathrm{~W} / \mathrm{mK}$ (see Table 1 ).

4.5. CFD Porous Medium Methodology. For the porous medium approach the CFD model has a mass of cells representing the fluid inlet. This is followed by the porous medium cells, which are used to model fluid flow through porous medium. Full flow field predictions are possible with the porous medium approach because the resistance of the porous medium to flow is described by the expression

$$
\frac{\Delta P}{L}=-\alpha U_{s}^{2}-\beta U_{s}
$$

where the permeability coefficient values $\alpha$ and $\beta$ are assigned temperature-dependent values that describe the behaviour of a porous medium. High values of $\alpha$ and $\beta$ preclude flow at right angles to the porous medium. 


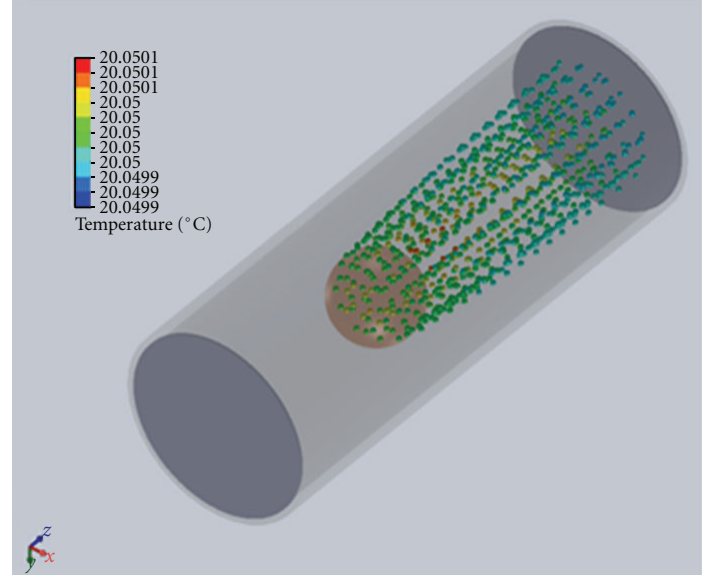

(a)

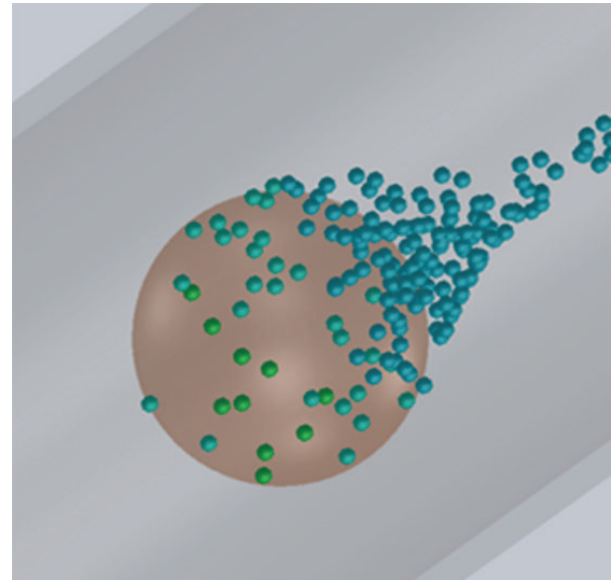

(b)

FIGURE 11: simulation of water vapour desorption on porous silica gel. To simulate desorption of water vapour we created a function called desorption that could take several inputs (material, fluid, pressure, flow rate, and whatever factors that would affect silica gel desorption of water vapour (see Figure 11).

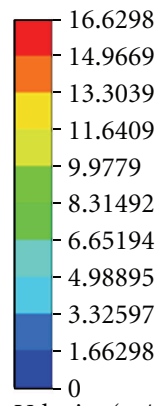

Velocity $(\mathrm{m} / \mathrm{s})$

Vector plot: velocity $(\mathrm{m} / \mathrm{s})$

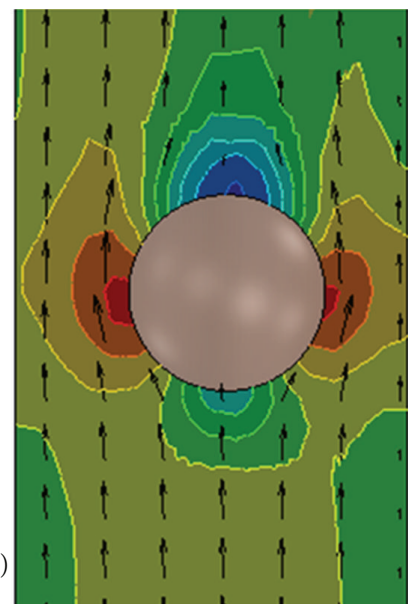

FIGURE 12: Velocity vectors profile vector plot of the water vapour velocity field around a single silica gel. The inlet velocity is $5 \mathrm{~m} / \mathrm{s}$ and the velocity field plotted in the plane passing through the centre of the particle.

Upstream and downstream of the water vapour flow field are solved using the usual Reynolds averaged Navier-Stokes methodology.

4.6. Boundary Conditions Used in Simulation. The boundary conditions determine the flow and thermal variables on the boundaries of the physical model. There are a number of boundary conditions:

(i) flow inlet and exit boundaries: pressure inlet, velocity inlet, pressure outlet,

(ii) wall, repeating, and limit boundaries: wall, symmetry,

(iii) internal fluid, solid,

(iv) internal face boundaries: porous, wall, interior.
In our model we use a velocity inlet at the flow inlet of the adsorption bed, this boundary condition defines a flow velocity at the inlet of the bed; The flow exit boundary is defined as a pressure outlet; the outlet pressure is defined as atmospheric pressure. The bed and packing interior are defined as boundaries. The wall boundaries separate the fluid zone, vapour, in between the silica gel particles from the wall zones [5, 9-11].

With the determination of the boundary conditions, the physical model has been defined, and a numerical solution can be provided. It was then necessary to determine how the solution will be established. This was done by setting the iteration parameters. With all boundary conditions defined, a number of additional parameters and solving schemes were selected.

An initial condition was assigned to the model and was used to help speed the convergence of the computation. The computation is an iterative process that solves the governing equations for flow and energy in each simulated cell. Depending on the complexity of the model and the computer resources available, CFD simulation can take anywhere from minutes to days [12]. The results of the simulation can be viewed and manipulated with postprocessing software once the simulation has converted to a solution.

4.7. CFD Mesh Generation. One of the most important parts of this CFD simulation was the module mesh creation. The mesh establishes the accuracy of the simulation which has to be chosen with enough detail to describe the simulation processes accurately and with a degree of coarseness that enables solution within an acceptable amount of time. When an optimal density has been found refining this will increase the model size without displaying more flow details. When it is coarseneds the mesh will obscure, possibly essential, part of the flow detail. The mesh determines a large part of creating an acceptable simulation. 
4.8. The CFD Mesh Density. For this study we focused mainly on maintaining a $3 \mathrm{D}$ simulation that described the physical model accurately and was able to handle the flow specifics of the silica gel geometry.

The mesh densities were varied to establish the optimal mesh density, describing the flow characteristics and limiting the calculation times.

The initial mesh was specified in the following stages.

(1) Specifying an automatic initial mesh, so all the following specifications consist in changing the default values of its parameters.

(2) Specifying a basic mesh consisting of nearly uniform cells.

(3) Refinement of the basic mesh to capture the relatively small silica gel porous features to resolve the small porous features in contact with fluid.

(4) Refinement of the basic mesh to resolve the solid/ fluid interface as well as porous/solid, fluid/porous interfaces.

(5) Specifying other initial meshes in local regions (solid and/or fluid) to better resolve the model-specific geometry and/or flow (and/or heat transfer in solids) peculiarities in these regions.

4.9. Postprocessing the Simulation Data. When the simulation has converged, the last data set is stored as a final solution. This data set has a record of the status of all elements in the model, temperature, densities, pressures, flow aspects, and so forth. To be able to interpret the data, it needs to be ordered and reduced to comprehensible sizes [2]. This displaying of the data is called postprocessing and makes it possible to compare the different simulations with each other and with external data. There were as many ways of displaying the data as there were data points, so it was important to select the data representation that was required for the desired data comparison. Some of the standard options available are contour plots and velocity vector plots. Contour plots will give a plot in the defined data point collection; this can be a plane or a volume of contours of another variable. For example, a plane can be defined as a constant $x$ coordinate plane $(y-z$ plane); we can then make a contour plot showing temperature contours in this plane. In the same plane a velocity contour plot can be made showing absolute velocities of the fluid in the defined plane. Other variables that can be used for contour plots are magnitude of velocity components, turbulence components, pressure, and so forth. Velocity vector plots can be made to get an insight into the flow patterns in the overall geometry or details at specific locations [7, 12-14].

4.10. Fluid Flow Fundamentals. For iteration CFD solvers, use generalized fluid flow and energy balances based on the Navier Stokes equations. The balances are generalized so that the user can influence which elements are added in the balance and which are not. The number of balances to be solved is also user defined; it can be advantageous to not solve all balances initially. The generalized balances that are used by the Flow simulation commercial CFD package are the Navier Stokes equations for conservation of mass and momentum, when it is set to calculate laminar flow without heat transfer. Additional equations are solved for heat transfer, species mixing or reaction or $\kappa$ and $\varepsilon$ for turbulent cases $[2,4,15]$.

\section{CFD Results and Discussion}

Figure 8 shows the adsorption curves for water adsorption on one granular size of silica gel; this curve was compared to Fuji data for smaller silica gel granules.

Figure 8 is an adsorption isotherm plot comparison between CFD simulation and the experimental results on silica gel size $3.5 \mathrm{~mm}$.

Figure 9 is an adsorption isotherm plot comparison between CFD simulation and the experimental results on silica gel size $5 \mathrm{~mm}$.

5.1. Water Vapour Adsorption CFD Simulation. The CFD simulation determines the distribution of water vapour molecules in the flow vapour phase and the adsorption of adsorbed vapour molecules on the silica gel surfaces. Figure 10 shows a silica gel porous media with a typical adsorption of water vapour molecules; this simulation was developed through a user-defined function developed to solve the Brunauer, Emmett, and Teller (BET) equation for both adsorption and desorption processes.

The developed model was used to determine the adsorption capacity of two different sizes of silica gel granules, namely, $3.5 \mathrm{~mm}$ and $5 \mathrm{~mm}$ as a function of time at different operating temperatures.

Figure 10 shows the water vapour being adsorbed onto the single silica gel. The porous media capabilities of CFD model determine the distribution of water vapour molecules in the flow vapour phase and the adsorption of adsorbed vapour molecules on the silica gel surfaces.

\subsection{CFD Simulation of Desorption of Water Vapour from Silica} $\mathrm{Gel}$. When attempting to simulate the desorption of water vapour from silica gel, it was necessary to create a userdefined function (UDF). The major challenge in the use of CFD simulation of the desorption of water vapour from silica gel is the complex interconnected void space silica gel has. In traditional 2D mathematical approaches the engineers use to largely ignoring this fact. In CFD simulation this is not ignored; it is modeled into the $3 \mathrm{D}$ model of the porous media. In order to simulate desorption, we had to create a function called desorption that would take several inputs (material, fluid, pressure, flow rate, and whatever factors affecting the silica gel calculations; see Figure 12 where there is a simulation of the water vapor desorption).

5.3. Velocity Profiles. To study the velocity distribution in an adsorption silica gel packed bed section cuts were made along the packed bed to generate velocity vector plots. Velocity profiles were also observed in the near-wall region of the 
modelled arrangements. As expected, in all of the cases analyzed, flow channelling took place near the wall and inside the bed, due to the presence of constrained flow areas. Strong radial flow from the middle to the wall was also noticeable. Due to the channelling of the flow (strong axial flow and reduced radial flow) at the wall, the local radial heat transfer rate decreases, causing the well-known.

\section{Conclusions}

A CFD model was developed for simulating the adsorpitivity of water vapour on silica gel granules and used to study the effect of granule sizes indicating that reducing the granule size increases the adsorpitivity. We used a thermogravimetric (TGA) to make some experimental data to be used in the CFD module; we compare our measurements with the CFD simulation. A good agreement was found with CFD and experimental data. In conclusion to this study, it is thought that using CFD could provide useful information for the design of adsorption cooling systems and better predict the system performance.

\section{References}

[1] K. E. Bertil Andersson, "Pressure drop in ideal fluidization," Chemical Engineering Science, vol. 15, no. 3-4, pp. 276-297, 1961.

[2] S. Ergun, "Fluid flow through packed columns," Chemical Engineering Progress, vol. 48, pp. 89-94, 1952.

[3] O. Molerus and J. Schweinzer, "Resistance of particle beds at Reynolds numbers up to $\mathrm{Re} \approx 104$," Chemical Engineering Science, vol. 44, no. 5, pp. 1071-1079, 1989.

[4] A. G. Dixon and D. L. Cresswell, "Effective heat transfer parameters for transient packed-bed models," AIChE Journal, vol. 32, no. 5, pp. 809-819, 1986.

[5] M. Rahimi and M. Mohseni, "CFD modeling of the effect of absorbent size on absorption performance of a packed bed column," Korean Journal of Chemical Engineering, vol. 25, no. 3, pp. 395-401, 2008.

[6] J. L. Steger and J. A. Benek, "On the use of composite grid schemes in computational aerodynamics," Computer Methods in Applied Mechanics and Engineering, vol. 64, no. 1-3, pp. 301-320, 1987.

[7] Akira Akahira, K. C. Amanul Alam, Yoshinori Hamamoto, Atsushi Akisawa, and Takao Kashiwagi, "Experimental investigation of mass recovery adsorption refrigeration cycle," International Journal of Refrigeration, vol. 28, no. 4, pp. 565$572,2005$.

[8] K. S. Chang, M. T. Chen, and T. W. Chung, "Effects of the thickness and particle size of silica gel on the heat and mass transfer performance of a silica gel-coated bed for air-conditioning adsorption systems," Applied Thermal Engineering, vol. 25, no. 14-15, pp. 2330-2340, 2005.

[9] K. S. Chang, M. T. Chen, and T. W. Chung, "Effects of the thickness and particle size of silica gel onthe heat and mass transfer performance of a silica gel-coated bed for air-conditioning adsorption systems," Applied Thermal Engineering, vol. 25, no. 14-15, pp. 2330-2340, 2005.

[10] May-Fun Liou, A numerical study of transport phenomena in porous media, Ph.D. thesis, Case Western Reserve University, 2005.
[11] G. E. Mueller, "Radial void fraction distributions in randomly packed fixed beds of uniformly sized spheres in cylindrical containers," Powder Technology, vol. 72, no. 3, pp. 269-275, 1992.

[12] A. Kopanidis and A. Theodorakakos, "Numerical simulation of fluid flow and heat transfer with direct modelling of microscale geometry," in Proceedings of the 5th European Thermal-Sciences Conference, The Netherlands, 2008.

[13] A. V. Anikeenko, N. N. Medvedev, M. K. Kovalev, and M. S. Melgunov, "Simulation of gas diffusion in porous layers of varying structure," Journal of Structural Chemistry, vol. 50, no. 3, pp. 403-410, 2009.

[14] D. C. Wang, J. Y. Wu, Z. Z. Xiaa, H. Zhaia, R. Z. Wanga, and W. D. Dou, "Study of a novel silica gel-water adsorption chiller. Part II. Experimental study," International Journal of Refrigeration, vol. 28, no. 7, pp. 1084-1091, 2005.

[15] F. Augier, C. Laroche, and E. Brehon, "Application of computational fluid dynamics to fixed bed adsorptioncalculations: effect of hydrodynamics at laboratory and industrial scale," Separation and Purification Technology, vol. 63, no. 2, pp. 466474, 2008.

[16] F. Augier, C. Laroche, and E. Brehon, "Application of computational fluid dynamics to fixed bed adsorption calculations: effect of hydrodynamics at laboratory and industrial scale," Separation and Purification Technology, vol. 63, no. 2, pp. 466474, 2008. 

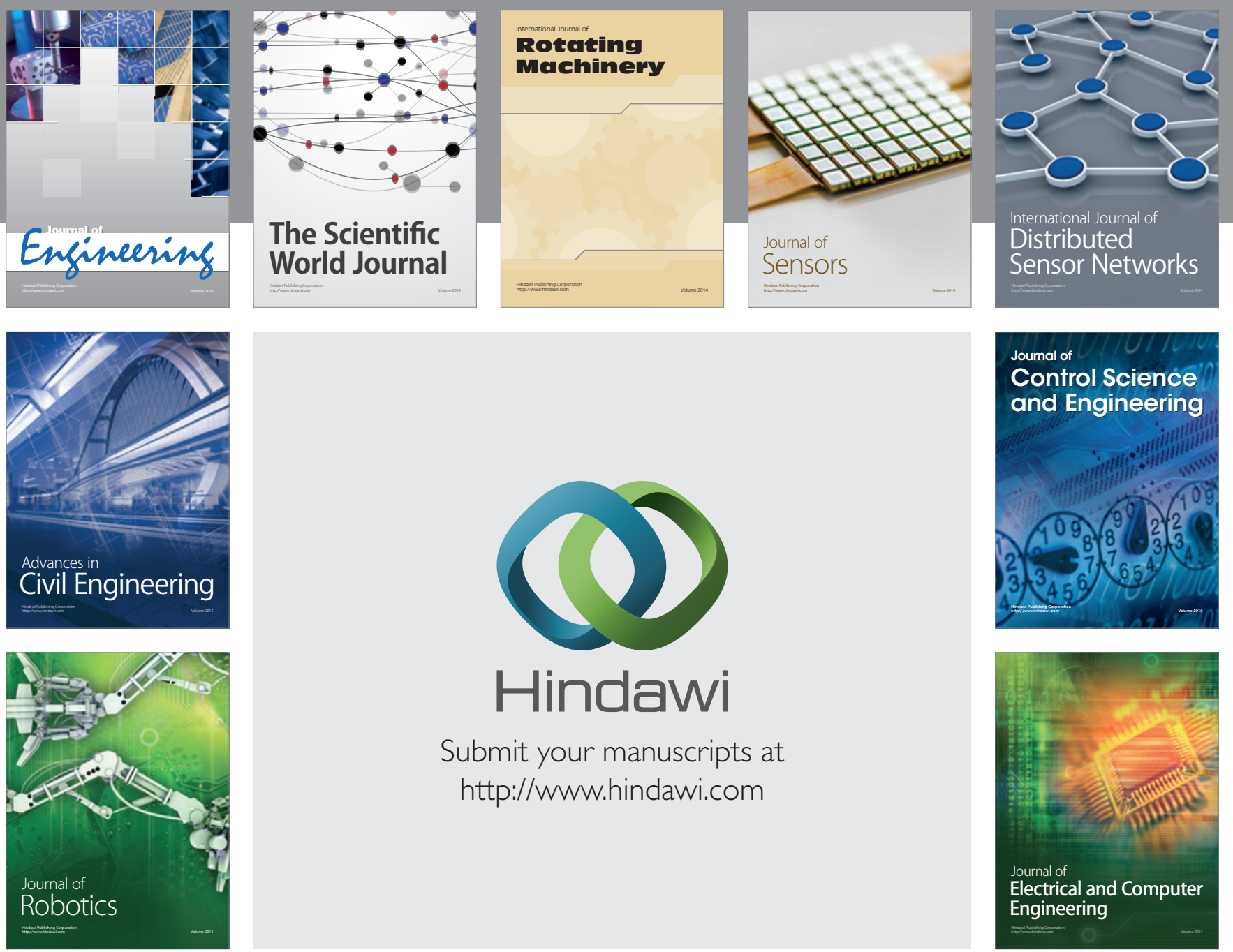

Submit your manuscripts at

http://www.hindawi.com
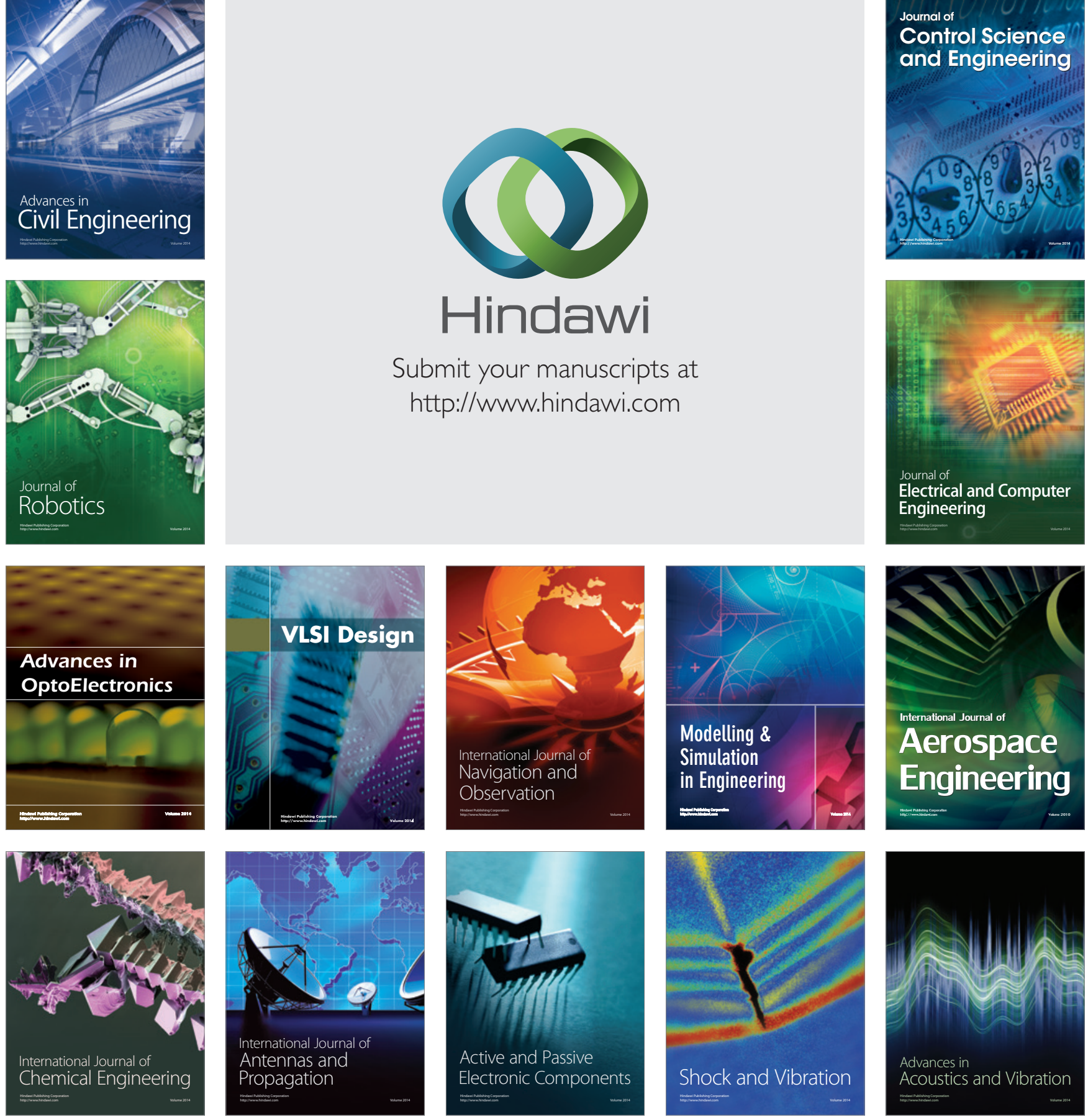